Ger J Exerc Sport Res 2021 · 51:1-3 https://doi.org/10.1007/s12662-020-00702-6 Eingegangen: 4. Dezember 2020

Angenommen: 15. Dezember 2020

Online publiziert: 26. Januar 2021

c c Der/die Autor(en) 2021

\section{Erwiderung}

Zum Leserbrief von Bloch, W., Hirschmüller, A., Mayer, F. et al. Leserbrief zu Thieme, L. Jung stirbt, wen die Götter lieben? Ger J Exerc Sport Res 50, 280-296 (2020). https://doi.org/10. 1007/s12662-020-00654-x. Ger J Exerc Sport Res 50, 477-479 (2020). https://doi.org/10. 1007/s12662-020-00681-8

\section{Originalbeitrag}

Thieme, L. Jung stirbt, wen die Götter lieben? Ger J Exerc Sport Res 50, 280-296 (2020). https://doi.org/10.1007/s12662-020-00654$\mathrm{x}$.

Es kommt nicht oft vor, dass ein Gremium einer Fachgesellschaft zu vorgelegten Ergebnissen aus anderen Disziplinen der Sportwissenschaft Stellung nimmt (Bloch et al., 2020). Umso mehr bedanke ich mich für die Würdigung des Beitrages zum Mortalitätsrisiko deutscher Olympiateilnehmerinnen und -teilnehmer (Thieme, 2020), vor allem aber für die konstruktiven Hinweise und Argumente, die insbesondere methodische Unsicherheiten sowie die Interpretation der Ergebnisse betreffen.

Der Leserbrief enthält eine ganze Reihe von Positionen und Argumenten, die sich mit denen im Beitrag vollumfänglich decken. Insbesondere sind weitere Analysen unter Einbindung zusätzlicher Faktoren notwendig, um Ursache-Wirkungs-Zusammenhänge zum Mortalitätsrisiko von Spitzensportlerinnen und -sportlern zu identifizieren. Mit Blick auf den Forschungsstand zur

Lutz Thieme (i)

RheinAhrCampus Remagen, Hochschule Koblenz, Remagen, Deutschland

\title{
Replik zum Leserbrief des Wissenschaftsrates der DGSP zum Beitrag "Jung stirbt, wen die Götter lieben?"
}

Erklärung von Lebensspannen sollten neben medizinischen Faktoren dabei auch psychologische und sozioökonomische Faktoren Beachtung finden. Ein derartiges erstes integratives Modell zur Erklärung der Lebensspanne schlagen Thieme und Fröhlich (2020) vor. Ebenso ist der Forderung der Autoren nach präventiv-sportmedizinischen Untersuchungen auch nach Karriereende zuzustimmen. Zwar kann - worauf die Leserbriefautoren völlig zu Recht hinweisen - eine endgültige Aussage über die Mortalität einer Gruppe im Vergleich zu einer anderen erst dann getroffen werden, wenn alle Personen in beiden Gruppen verstorben sind. Aus diesem Grunde sind Mortalitätsstudien per se immer vorläufig und weisen höhere Unsicherheiten auf, je jünger die Risikogruppe ist und je weniger Todesfälle in dieser Gruppe zu beklagen sind. Daher wird in der besprochenen Studie u.a. auch nur äußerst zurückhaltend über das Risiko der Olympiateilnehmerinnen und -teilnehmer $\mathrm{ab}$ einer erstmaligen Olympiateilnahme 1992 für das wiedervereinigte Deutschland berichtet. Angesichts dieser prinzipiellen methodischen Grenzen stellen Folgerungen aus Mortalitätsstudien für die vom untersuchten Risiko betroffenen Gruppen immer eine besondere inhaltliche und zeitliche Herausforderung dar. Welches $\mathrm{Maß}$ an Evidenz muss erreicht werden (z. B. hypothesenprüfend, praktisch, klinisch etc.), um die aktuelle Risikogruppe und die in die Risikogruppe strebenden Menschen über mutmaßliche Risikofak- toren zu informieren, dem statistischen Risiko vorzubeugen oder gar mit Interventionen vor etwaigen Folgen $\mathrm{zu}$ schützen? Solche Fragen können für den Leistungssport wohl nur vergleichbar zur Anwendung des Vorsorgeprinzips in anderen gesellschaftlich relevanten Lebensbereichen beantwortet werden, womit eine Diskussion der Folgen erst nach dem Tod aller Personen der Risikogruppe nicht vereinbar sein dürfte.

Mit den Leserbriefschreibern stimme ich jedenfalls darin überein, dass die vorgelegten Daten die Annahme einer konkreten und generellen Lebenszeitverkürzung für alle deutschen Teilnehmer am Spitzensport derzeit nicht rechtfertigen (Bloch et al., 2020, S. 478). Die vorgelegten Daten zeigen jedoch auch deshalb Forschungsbedarf an, da die internationale Befundlage durchaus kritischer beurteilt werden kann, als dies die Leserbriefautoren tun. Beispielsweise umfasst die von ihnen genannte Studie von Clarke et al. (2015) Daten der olympischen Medaillengewinner von 1896 bis 2010, betrachtet Deutschland wegen der beiden Weltkriege jedoch erst ab 1950 (1906 Medaillengewinner abzüglich 214 wegen fehlender Daten), belässt aber u. a. Frankreich, Italien und die USA im Sample. Studien mit derart langen Untersuchungszeiträumen laufen Gefahr, die mit Kriegen verbundenen unterschiedlichen Mortalitätsrisiken für körperlich Tüchtige und verschiedene soziale Gruppen (vgl. dazu die Diskussion der Überlebenswahrscheinlichkeiten in Ausnahmesituationen z.B. bei Frey, 
Tab. 1 Cox-Regression zum Vergleich der Mannschaften der BRD und der DDR

\begin{tabular}{|c|c|c|c|c|c|c|c|c|}
\hline & \multirow[t]{2}{*}{ B } & \multirow[t]{2}{*}{ SE } & \multirow[t]{2}{*}{ Wald } & \multirow[t]{2}{*}{ Df } & \multirow[t]{2}{*}{$\begin{array}{l}\text { Signifi- } \\
\text { kanz }\end{array}$} & \multirow[t]{2}{*}{$\operatorname{Exp}(B)$} & \multicolumn{2}{|c|}{$\begin{array}{l}95 \% \text { Konfidenzinter- } \\
\text { vall für } \operatorname{Exp(B)}\end{array}$} \\
\hline & & & & & & & Untere & Obere \\
\hline Geschlecht & 0,752 & 0,180 & 17,474 & 1 & 0,000 & 2,122 & 1,491 & 3,020 \\
\hline $\begin{array}{l}\text { Alter bei erstma- } \\
\text { liger Olympiateil- } \\
\text { nahme }\end{array}$ & 0,019 & 0,009 & 4,331 & 1 & 0,037 & 1,020 & 1,001 & 1,038 \\
\hline $\begin{array}{l}\text { Olympische Som- } \\
\text { mer- oder Winter- } \\
\text { spiele }\end{array}$ & $-0,157$ & 0,127 & 1,514 & 1 & 0,218 & 0,855 & 0,666 & 1,097 \\
\hline $\begin{array}{l}\text { Anzahl an Teilnah- } \\
\text { men }\end{array}$ & $-0,040$ & 0,082 & 0,236 & 1 & 0,627 & 0,961 & 0,819 & 1,128 \\
\hline $\begin{array}{l}\text { Anzahl an Medail- } \\
\text { len }\end{array}$ & 0,201 & 0,061 & 11,053 & 1 & 0,001 & 1,223 & 1,086 & 1,377 \\
\hline $\begin{array}{l}\text { Mannschafts- } \\
\text { sportarten }\end{array}$ & - & - & 0,544 & 2 & 0,762 & - & - & - \\
\hline Einzelsportarten & 0,080 & 0,126 & 0,403 & 1 & 0,525 & 1,083 & 0,846 & 1,386 \\
\hline Mixsportarten & 0,007 & 0,159 & 0,002 & 1 & 0,966 & 1,007 & 0,737 & 1,376 \\
\hline $\begin{array}{l}\text { Jahr der erstmali- } \\
\text { gen Olympiateil- } \\
\text { nahme }\end{array}$ & $-0,029$ & 0,007 & 16,029 & 1 & 0,000 & 0,971 & 0,958 & 0,985 \\
\hline BRD versus DDR & 0,437 & 0,112 & 15,152 & 1 & 0,000 & 1,548 & 1,242 & 1,929 \\
\hline
\end{tabular}

Savage, Schmidt, \& Torgler, 2011; sowie Diekmann, 2012) und sich wandelnde schichtspezifische und sozioökonomisch moderierte Zugänge $\mathrm{zu}$ verschiedenen Sportarten, zum Leistungssportsystem und zur Sportförderung zu inkludieren. Hinzu kommt eine fragile Statistik der Sterberaten in der Gesamtbevölkerung, die über den gesamten Untersuchungszeitraum und nicht für alle Länder mit heutigen Standards $\mathrm{zu}$ vergleichen ist. Derartige Effekte können dann dazu führen, dass die 341 männlichen Olympiasieger der Jahre 1896-2016 im Ringen statistisch im Durchschnitt 19,1 Jahre länger leben als die Gesamtbevölkerung des gleichen Geburtsjahrgangs im Herkunftsland (Keller, 2019). Werden die Beobachtungszeiträume verkürzt oder kategorisiert, sinkt mit der Größe der Risikogruppe und der Todesfälle die $\mathrm{Zu}$ verlässigkeit der statistischen Verfahren bei steigendem Risiko der Produktion von Zeitintervalleffekten. Methodische Unsicherheiten sind demzufolge integraler Bestandteil jeder wahrscheinlichkeitsbasierten Mortalitätsstudie.

Bedeutsam für den Vergleich des Mortalitätsrisikos zwischen einer Untersu- chungsgruppe und der Gesamtbevölkerung ist zudem der Umgang mit der Säuglings- und Kindersterblichkeit, die gegenüber den nachfolgenden Lebensjahren deutlich erhöht ist. In den meisten Studien begnügen sich die Autoren mit dem Hinweis auf die Herkunft der Mortalitätsraten (z. B. Clarke et al., 2015, S. 899; Marijon et al., 2013, S. 3146; Takeuchi et al., 2019, S. 2). Daher ist davon auszugehen, dass in den bisherigen Studien das Mortalitätsrisiko der Gesamtbevölkerung von Geburt an als Vergleich herangezogen und entsprechende Vergleichsgruppen nach Geschlecht und Geburtsjahr zur Gruppe der jeweils untersuchten Sportlerinnen und Sportler konstruiert wurden. Somit würden die relativ hohen Sterblichkeitsraten der Kindheit in der Gesamtbevölkerung in den Vergleich mit einer Risikogruppe einfließen, die diese Periode bereits überlebt hat. Im Gegensatz dazu wurde in der vorgelegten Studie das Mortalitätsrisiko der Gesamtbevölkerung erst ab dem 14. Lebensjahr berücksichtigt.

Während keine generelle Lebenszeitverkürzung für alle deutschen Spitzensportlerinnen und Spitzensportler kon- statiert werden kann und auch das Risiko der aktuellen Generation der deutschen Olympiateilnehmerinnen und -teilnehmer aufgrund der schmalen Datenbasis nur sehr eingeschränkt $\mathrm{zu}$ beurteilen ist, stellt sich die Datenlage auch unter Berücksichtigung der methodischen Unsicherheiten beim Vergleich zwischen den Olympiateilnehmerinnen und -teilnehmern aus Ost und West gefestigter dar, als dies die Leserbriefschreiber annehmen. Wie von der Autorin und den Autoren des Leserbriefes zutreffend angemerkt, kontrollieren die zum Vergleich zwischen beiden Gruppen verwendete Wilcoxon-Gehan-Statistik sowie der Log-Rank-Test die möglichen Wirkungen unterschiedlicher Einflussfaktoren wie beispielsweise die Verteilung der Geschlechter und die Medaillenanzahl als Proxy für den sportlichen Erfolg nicht. Die nachfolgende Anwendung der Cox-Regression dürfte angesichts der in das Modell eingefügten zeitinvariablen Einflussgrößen methodisch wenig problematisch sein. Zudem kreuzen sich die Kaplan-Meier-Kurven nicht (Zwiener, Blettner, \& Hommel, 2011). Cox-Regressionen adjustieren wie andere Regressionsverfahren auch die verschiedenen unabhängigen Variablen in ihrer Wirkung auf die abhängige Variable (Ziegler, Lange, \& Bender, 2004). Führt man eine Cox-Regression unter Einbezug aller deutschen Olympiateilnehmerinnen und Teilnehmer durch, dann ist die Zuordnung zu einer der drei Olympiamannschaften (Ost, West, wiedervereinigtes Deutschland) der zweitstärkste Risikofaktor (Thieme, 2020, Tab. 4). Allerdings schließen die 95\% Konfidenzintervalle für das Hazard-Ratio für die alte Bundesrepublik (68\% bzw. 394\%) ebenso wie für die DDR (43\% bzw. $254 \%$ ) das auf das wiedervereinigte Deutschland bezogene Hazard-Ratio ein. Es ist daher zunächst nicht zu entscheiden, welche der drei Gruppen einem höheren Risiko ausgesetzt ist (Thieme, 2020, S. 289). Exkludiert man nun jedoch die Athletinnen und Athleten, die erstmals 1992 für das wiedervereinigte Deutschland an Olympischen Spielen teilgenommen haben ( $n=2325$; sieben Todesfälle), dann dürften die Ergebnisse einer Cox-Regression für die verbleibende Gruppe aus 
Teilnehmerinnen und Teilnehmern für die ehemalige Bundesrepublik $(n=2107$; 275 Todesfälle) und die DDR ( $n=1634$; 118 Todesfälle) vertrauenswürdig sein, da sowohl die Zahl der Gruppe als auch die Todesfälle in den Subgruppen genügend groß sind. Zudem geht mit dem Jahr der erstmaligen Olympiateilnahme ein Faktor in die Cox-Regression ein, der als Proxy für die im Zeitverlauf gestiegene Lebenserwartung interpretiert werden kann. Die Ergebnisse dieser CoxRegression werden im Originalbeitrag zwar verbal beschrieben (Thieme, 2020, S. 290), jedoch nicht vollständig und explizit berichtet. Dies soll an dieser Stelle nachgeholt werden (• Tab. 1).

Wie im Originalbeitrag ausgewiesen, ist die Zuordnung zur Mannschaft der alten Bundesrepubliknach dem Geschlecht der zweithöchste Risikofaktor und hoch signifikant. Das zugehörige Konfidenzintervall erstreckt sich von 124-192\%. Die Angehörigen der Olympiamannschaften der alten Bundesrepublik haben demnach gegenüber den Angehörigen der Olympiamannschaften der DDR ein zwischen 1,24- und 1,92-fach höheres Risiko, früher zu versterben. Selbstverständlich handelt es sich auch hierbei um einen vorläufigen, weiter $\mathrm{zu}$ eruierenden $\mathrm{Be}$ fund. Dennoch scheint mit dem Anliegen der Leserbriefschreiber, diesen Vergleich „nur mit allergrößter Vorsicht zu interpretieren“ (Bloch, et al., 2020, S. 478) vereinbar zu sein, derzeit von einem Überlebensvorteil für die Olympiateilnehmerinnen und -teilnehmer der DDR auszugehen.

Abschließend sei nochmals darauf verwiesen, dass es sich bei den angewandten statistischen Verfahren, wie beispielsweise dem Kaplan-Meier-Verfahren und den Cox-Regressionen, um Standardverfahren für diese Art von Problemstellung handelt, die (1) immer eine Adäquatheit voraussetzen, (2) methodischen Limitation unterliegen, (3) Wahrscheinlichkeitsaussagen machen, die (4) nur so gut sind, wie die zur Verfügung stehenden Daten und (5) eine hypothesenprüfende, praktische und klinische Relevanz besitzen, die nur theoretisch begründet werden können.

\section{Korrespondenzadresse

Lutz Thieme
RheinAhrCampus Remagen,
Hochschule Koblenz
Joseph-Rovan-Allee 2,
534242 Remagen,
Deutschland
thieme@rheinahrcampus.de

Funding. Open Access funding enabled and organized by Projekt DEAL.

Interessenkonflikt. L. Thieme gibt an, dass kein Interessenkonflikt besteht.

Open Access. Dieser Artikel wird unter der Creative Commons Namensnennung 4.0 International Lizenz veröffentlicht, welche die Nutzung, Vervielfältigung, Bearbeitung, Verbreitung und Wiedergabe in jeglichem Medium und Format erlaubt, sofern Sie den/die ursprünglichen Autor(en) und die Quelle ordnungsgemäß nennen, einen Link zur Creative Commons Lizenz beifügen und angeben, ob Änderungen vorgenommen wurden.

Die in diesem Artikel enthaltenen Bilder und sonstiges Drittmaterial unterliegen ebenfalls der genannten Creative Commons Lizenz, sofern sich aus der Abbildungslegende nichts anderes ergibt. Sofern das betreffende Material nicht unter der genannten Creative Commons Lizenz steht und die betreffende Handlung nicht nach gesetzlichen Vorschriften erlaubt ist, ist für die oben aufgeführten Weiterverwendungen des $\mathrm{Ma}$ terials die Einwilligung des jeweiligen Rechteinhabers einzuholen.

Weitere Details zur Lizenz entnehmen Sie bitte der Lizenzinformation auf http://creativecommons.org/ licenses/by/4.0/deed.de.

\section{Literatur}

Bloch, W., Hirschmüller, A., Mayer, F., Nieß, A., Meyer, T., Reinsberger, C., et al. (2020). Leserbrief zu Thieme, L. Jung stirbt, wen die Götter lieben? Ger J Exerc Sport Res 50, 280-296 (2020). https://doi.org/10.1007/s12662-020-00654-x. German Journal of Exercise and Sport Research, 50(4), 477-479.

Clarke, P.M., Walter, S. J., Hayen, A., Mallon, W. J., Heijmans, J., \& Studdert, D. M. (2015). Survival of the fittest: retrospective cohort study of the longevity of Olympic medallist in the modern era. British Journal of Sports Medicine, 49(13), 898-902.

Diekmann, A. (2012). Die Rolle sozialer Normen, der Situationsdefinition und sozialer Klassen beim Untergang der Titanic. Kölner Zeitschrift für Soziologie und Sozialpsychologie, 64(1), 175-184.

Frey, B. S., Savage, D. A., Schmidt, L. S., \& Torgler, B. (2011). Auswirkungen von Macht auf das Überleben in Extremsituationen: Ein Vergleich der Titanic und Lusitania Schiffskatastrophen. Kölner Zeitschrift für Soziologie und Sozialpsychologie, 63(2), 237-254.

Keller, K. (2019). Life expectancy of Olympic wrestling champions in comparison to the general population. Journal of Community health, 44(1), 61-67. https://doi.org/10.1007/s10900-0180553-6.
Marijon, E., Tafflet, M., Antero-Jacquemin, J., El Helou, N., Berthelot, G., Celermajer, D. S., et al. (2013). Mortality of French participants in the Tour de France (1947-2012). European Heart Journal, 34(40), 3145-3150. https://doi.org/10.1093/ eurheartj/eht347.

Takeuchi, T., Kitamura, Y., Sado, J., Hattori, S., Kanemura, Y., Naito, Y., et al. (2019). Mortality of Japanese Olympic athletes. BMJ Open Sport \& Excerise Medicine, 5(1), e653. https://doi.org/10.1136/ bmjsem-2019-000653.

Thieme, L. (2020). Jung stirbt, wen die Götter lieben? Zur Mortalität deutscher Olympiateilnehmer 1956 bis 2016. German Journal of Exercise and Sport Research, 50(2), 280-296. https://doi.org/ 10.1007/s12662-020-00654-x.

Thieme, L., \& Fröhlich, M. (2020). Do former elite athletes live longer? New evidence from German olympic athletes and a first model description. Frontiers in Sports and Active Living, 2, 588204. https://doi.org/10.3389/fspor.2020.588204.

Ziegler, A., Lange, S., \& Bender, R. (2004). Überlebenszeitanalysen: Die Cox-Regression. Deutsche Medizinische Wochenschrift, 129(503), 1-3.

Zwiener, l., Blettner, M., \& Hommel, G. (2011). Überlebenszeitanalysen. Deutsches Ärzteblatt, 108(10), 163-169. 